\title{
Tailoring Polymer Dispersity by Controlled Radical Polymerization: A Versatile Approach
}

Richard Whitfield, ${ }^{\dagger}$ Kostas Parkatzidis, ${ }^{\dagger}$ Nghia P. Truong,,${ }^{\dagger}+$ Tanja Junkers* ${ }^{\S}$ and Athina Anastasaki*ं

$\dagger$ - Laboratory of Polymeric Materials, Department of Materials, ETH Zurich, Vladimir-Prelog-Weg 5, Zurich, Switzerland.

\$ - Monash Institute of Pharmaceutical Sciences, Monash University, 399 Royal Parade, Parkville, Victoria 3152, Australia

$\S$ - Polymer Reaction Design Group, School of Chemistry, Monash University, VIC 3800 Melbourne, Australia

*Correspondence: Athina.anastasaki@ mat.ethz.ch, tanja.junkers@ monash.edu

\section{ABSTRACT}

Dispersity $(\bigoplus)$ can significantly affect polymer properties and is a key parameter in materials design; however, current methods do not allow for the comprehensive control of dispersity. They are limited in monomer scope, may require the use of flow-based systems and/or additional reagents (e.g. termination agents or co-monomers), and are often accompanied by multimodal molecular weight distributions, low initiator efficiencies or poor end-group fidelity. Herein, we report a straightforward and versatile batch method based on reversible addition-fragmentation chain transfer (RAFT) polymerization which enables good control over $\oslash$ of a wide range of monomer classes, including acrylates, acrylamides, methacrylates and styrene. In addition, our methodology is compatible with more challenging monomers such as methacrylic acid, vinyl ketone and vinyl acetate. Control over $Ð$ is achieved by mixing two RAFT agents with sufficiently different transfer activities in various ratios, affording polymers with monomodal molecular weight distributions over a broad dispersity range $(\nexists \sim 1.09-2.10)$. Our findings were further supported by simulations through the use of deterministic kinetic modelling which was fully in line with our experimental data, further confirming the power of our methodology. The robustness of the concept is further 
demonstrated by the preparation of well-defined block copolymers via chain extension of all polymers regardless of the initial $Đ$.

\section{INTRODUCTION}

Controlled Radical Polymerization (CRP), also referred to as Reversible Deactivation Radical Polymerization (RDRP), has made an enormous impact on polymer science, as it provided the ability to regulate molecular weight, dispersity, composition, architecture and end-group fidelity of vinyl polymers. ${ }^{1-4}$ Nowadays, numerous CRP techniques are available with the two most dominant ones being Atom Transfer Radical Polymerization (ATRP) and Reversible Addition-Fragmentation chain-Transfer (RAFT) polymerization. ${ }^{5-8}$ These techniques have enabled a number of additional developments, including polymer selfassembly, microphase separation, bioconjugation and surface modification. ${ }^{9,10}$ Importantly, polymers made by CRP find use as emulsifiers, dispersants, rheology and surface modifiers, electrolytes and as nanocontainers for the encapsulation and delivery of active components in applications spanning across a range of markets including home care, beauty, health, paints, energy and electronics. ${ }^{10-12}$

Many of these applications and developments have emerged due to the vast majority of polymer chains produced by CRP having an active end-group, which can be exploited e.g. to form block copolymers. ${ }^{4}$ Apart from high end-group fidelity, special emphasis was given to the breadth of molecular weight distributions commonly measured as dispersity $(\nexists)$, a key parameter that determines the physical properties of a material. ${ }^{13,14}$ For years, most papers in controlled radical polymerization optimize conditions to obtain low $Ð$ polymers following a common misconception that high $Ð$ materials are less desirable and are often accompanied with lower end-group fidelity. ${ }^{4,15}$ However, recently it has been recognized that both high and low $Ð$ polymers exhibit unique properties and functions and as such being able to systematically tune the $\oslash$ can be advantageous for many applications as it can affect, among 
others, the processability of polymer melts, rheological properties and polymer selfassembly. ${ }^{16,17}$ In fact, in industry there are numerous examples of high $Ð$ polymers used with enhanced properties over their lower $Ð$ analogues. ${ }^{18-20}$

However, synthesizing polymers with higher $Ð$ has not been the focus of the vast majority of papers and it appears to violate the conventional principles of controlled radical polymerization, where high $Ð$ and high livingness are typically considered mutually exclusive (Quirk and Lee experimental criteria require low dispersity for a living system). ${ }^{21}$ Existing methods to obtain materials with tuneable $D$ are typically focusing on implementing engineering approaches to tackle the problem rather than designing new chemistries. For instance, the blending approach involves the synthesis of a number of polymers with different molecular weights followed by their mixing in predetermined ratios. ${ }^{22-26}$ This method can indeed allow for a broad range of $Ð$ to be obtained but it can be tedious and time-consuming due to the multiple synthesis and subsequent purifications required (as many as 20 polymers may need to be mixed), while it often results in multimodal molecular weight distributions. ${ }^{17,27}$ Elegant alternatives exploit the temporal regulation of initiation by using flow chemistry to slowly feed initiating species into the polymerization mixture. ${ }^{28-31}$ Such approaches rely on careful optimization of mixing/flow rates and also yield multimodal molecular weight distributions. ${ }^{32-35}$ A few examples of polymerization protocols where polymer $\oslash$ can be controlled by manipulation of the polymerization have recently been reported. For instance, Goto and co-workers utilized a small amount of a comonomer in reversible complexation mediated polymerization to modulate $Ð$ of methacrylates while Chiu's group reported the use of photochromic initiators in cationic polymerization. ${ }^{36,37}$ Matyjaszewski and co-workers also showed tailored $Ð$ in atom transfer radical polymerization (ATRP) by varying the catalyst concentration. ${ }^{38,39}$ Despite these significant advances, a major weakness of current strategies is that they are limited in monomer scope, as they typically work only for one particular 
monomer class. Other issues include polymers exhibiting low end-group fidelity and multimodal MWDs, limited demonstration of block copolymers, complex multicomponent systems, narrow range of accessible $Ð$ values, low initiator efficiency and the use of co-monomers, additives and pump-assisted procedures; the latter may be incompatible with surface polymerizations. ${ }^{22,31,32,36,39,40}$

Herein, we report a straightforward and versatile method which allows the systematic control of dispersity. By introducing a new concept in controlled radical polymerization, we exploited the mixing of RAFT agents with different chain transfer constants to tune the dispersity for a wide range of monomer classes, including acrylates, acrylamides, methacrylates (and methacrylic acid), styrene, vinyl ketone and vinyl acetate $(\nexists \sim 1.09-2.10)$. Our strategy afforded monomodal molecular weight distributions in all cases, and encompassed the preparation of a range of block copolymers, regardless of initial dispersity.

\section{RESULTS AND DISCUSSION}

We envisaged that we could overcome the aforementioned challenges by exploiting RAFT polymerization, a technique that has not yet been used to tune $Ð$ in batch. This is rather surprising, given that RAFT is one of the most versatile and robust RDRP techniques and is widely used to prepare a broad range of polymeric materials for diverse applications in various fields. ${ }^{5,9,41-44}$ In a conventional (i.e. thermal) RAFT polymerization, two components are essential: a free radical initiator to continuously supply radicals and a chain transfer agent (CTA) to mediate the equilibrium/exchange between dormant and active species. The latter is typically a thio-carbonylthio-based compound with the general structural formula $\mathrm{Z}-\mathrm{C}(=\mathrm{S})-\mathrm{S}-\mathrm{R}$. To change $Ð$, we initially considered increasing the concentration of the radical initiator azobisisobutyronitrile (AIBN), but this strategy was abandoned, as it would induce more termination and hence yield a polymer of very limited use. In theory, $Ð$ can be also varied by selecting individual CTAs with subtly different activities (different rate constant ratios of 
chain transfer to propagation), as predicted based on an equation proposed by Mueller et al. (i.e., one CTA would yield $Ð \sim 1.1$, another $Ð \sim 1.4$, a third $\oslash \sim 1.6$, etc.). ${ }^{45}$ However, this theoretical approach would require the specific design and synthesis of many different RAFT agents depending on the monomer class and targeted $\oslash$, followed by time-consuming and extensive kinetic optimizations for each new RAFT agent, thus making such approach impractical. Instead, we hypothesized that $Ð$ could be efficiently tuned by mixing two RAFT agents of notably different activities. This would reduce the number of CTAs required to just two, while allowing access to a much greater range of dispersity values. To the best of our knowledge, the concept of mixing RAFT agents prior to polymerization has not been implemented before and can be summarized in Fig. 1.

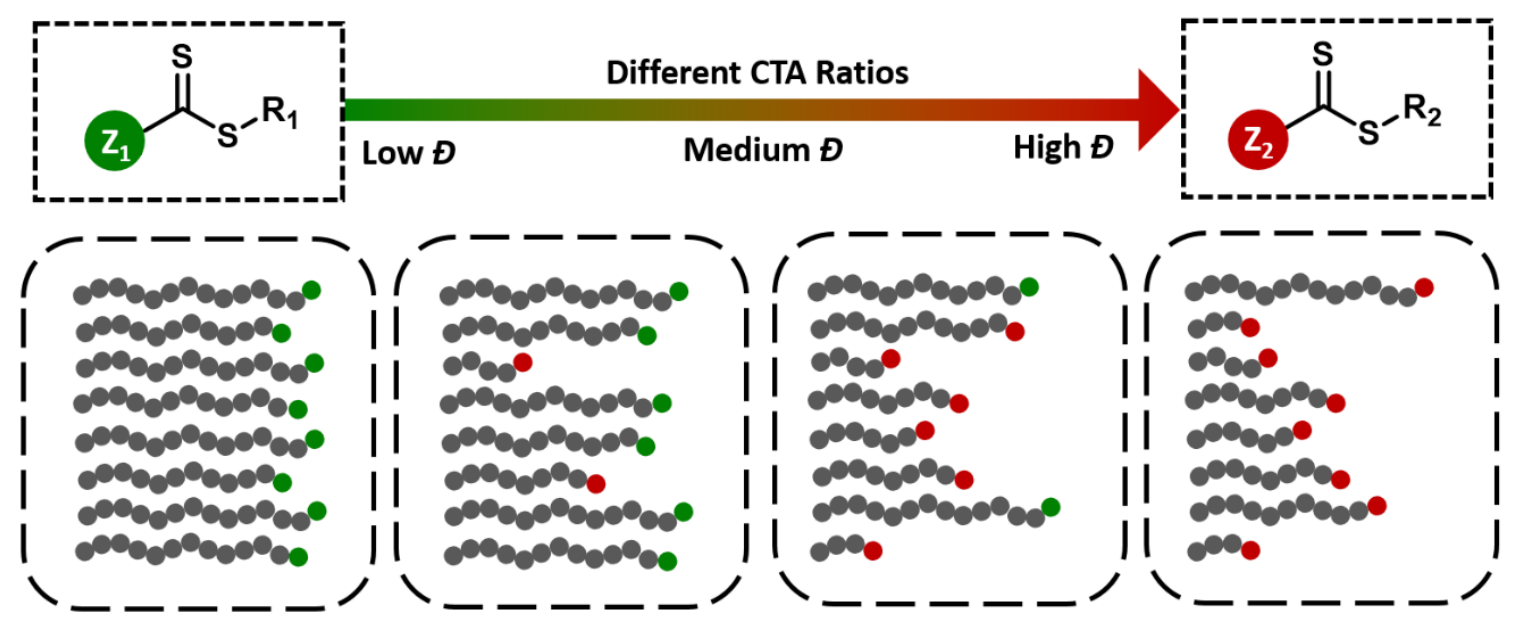

Fig. 1: Schematic representation of mixing chain transfer agents to tune polymer dispersity.

To investigate this hypothesis, initial experiments involved methyl methacrylate (MMA) as the monomer, AIBN as the free radical initiator and a mixture of two RAFT agents, of which one would afford good control over the polymerization and low $Ð$ (a CTA with a high activity or a high macroradical addition rate), and the second one would yield broader, yet monomodal MWDs (a CTA with lower efficiency or lower macroradical addition rate). 2cyanoprop-2-yl dithiobenzoate (CTA 1) was selected as a high-activity CTA for the polymerization of methacrylates. ${ }^{46-48}$ Upon polymerizing MMA, well-defined PMMA could 
be obtained with $M_{\mathrm{n}}=25300 \mathrm{~g} \mathrm{~mol}^{-1}\left(M_{\mathrm{w}}=28600 \mathrm{~g} \mathrm{~mol}^{-1}\right)$ and $Ð \sim 1.13$ (Fig. 2a and Supplementary Fig. 1 and Supplementary Table 1, Entry 5). In order to increase $\oslash$ while maintaining monomodal MWDs, we envisioned that a suitable second CTA with a much lower (yet still sufficient) macroradical addition rate than CTA 1 was needed. Unfortunately, the selection of such CTAs has not been the focus of literature and therefore required additional investigation. The activity of a RAFT CTA can be tuned by careful selection of the Z- and Rgroups. For instance, 2-cyano-2-propyl dodecyl trithiocarbonate (CTA 2) has a lower activity than CTA 1 because of the higher stability of the RAFT intermediate (alkylthio vs phenyl Zgroup). By using CTA 2, PMMA of slightly higher $Ð$ (1.23) was obtained, as anticipated (Supplementary Fig. 2a and Supplementary Table 2, Entry 1). When the even lower transfer activity 2-cyanobutan-2-yl 4-chloro-3,5-dimethyl-1H-pyrazole-1-carbodithioate (CTA 3) was used, ${ }^{49}$ PMMA with monomodal MWDs and a $Ð$ of 1.65 could be obtained (Supplementary Table 1, Entry 1). As expected, employing CTAs with much lower activity than CTA 3 (i.e., methyl 2-[methyl(4-pyridinyl)carbamothioylthio]propionate (CTA 5), 2-cyanopropan-2-yl Nmethyl- $N$-(pyridin-4-yl)carbamodithioate (CTA

6), cyanomethyl methyl(phenyl)carbamodithioate, $\quad$ 2-cyanobutan-2-yl $\quad 3,5$-dimethyl- $1 H$-pyrazole-1carbodithioate and methyl 2-(butylthiocarbonothioylthio)propanoate) resulted in notable lowMW tailing, very poor CTA efficiency and bimodal MWDs as evident by size exclusion chromatography (SEC, Supplementary Scheme 1, Supplementary Fig. 2 and Supplementary Table 2, Entries 2-6 $)^{49}$. As such, CTA 3 was selected as a suitable low-activity CTA to further study the polymerization of MMA. Pleasingly, by altering the ratio between the two RAFT agents (CTA 1 to CTA 3), the $Ð$ of PMMA could be successfully tuned between 1.13-1.65 (Fig. 2a, Supplementary Fig. 3 and Supplementary Tables 1, 3 Entries 1-5). Importantly, the MWDs remained monomodal throughout the polymerizations. 
In addition, when using the aforementioned mixtures of RAFT CTAs, high end-group fidelity could be demonstrated by the addition of a second aliquot of MMA. Indeed, upon using a PMMA macroCTA with $Ð \sim 1.4$, efficient chain extension was achieved $(\nexists=1.38$, Fig. $2 b$ \& Supplementary Fig. 4). This initial data suggests that our strategy of mixing RAFT CTAs can successfully tune $Ð$ yielding polymers with monomodal MWDs and high end-group fidelity. Importantly, any intermediate $Ð$ can be obtained by using a different mixing ratio. For instance, by choosing mixtures constituting of $80 \%$ CTA 1 and 20\% CTA 3, and $20 \%$ CTA 1 and $80 \%$ CTA 3, $Đ$ of 1.19 and 1.47 were obtained (Supplementary Fig. 5 and Supplementary Table 4), respectively, following a trend as shown in Supplementary Fig. 6. Overall, our approach of mixing two readily available RAFT agents is hence far simpler than designing and synthesizing specific RAFT agents for each desirable $\bigoplus$.
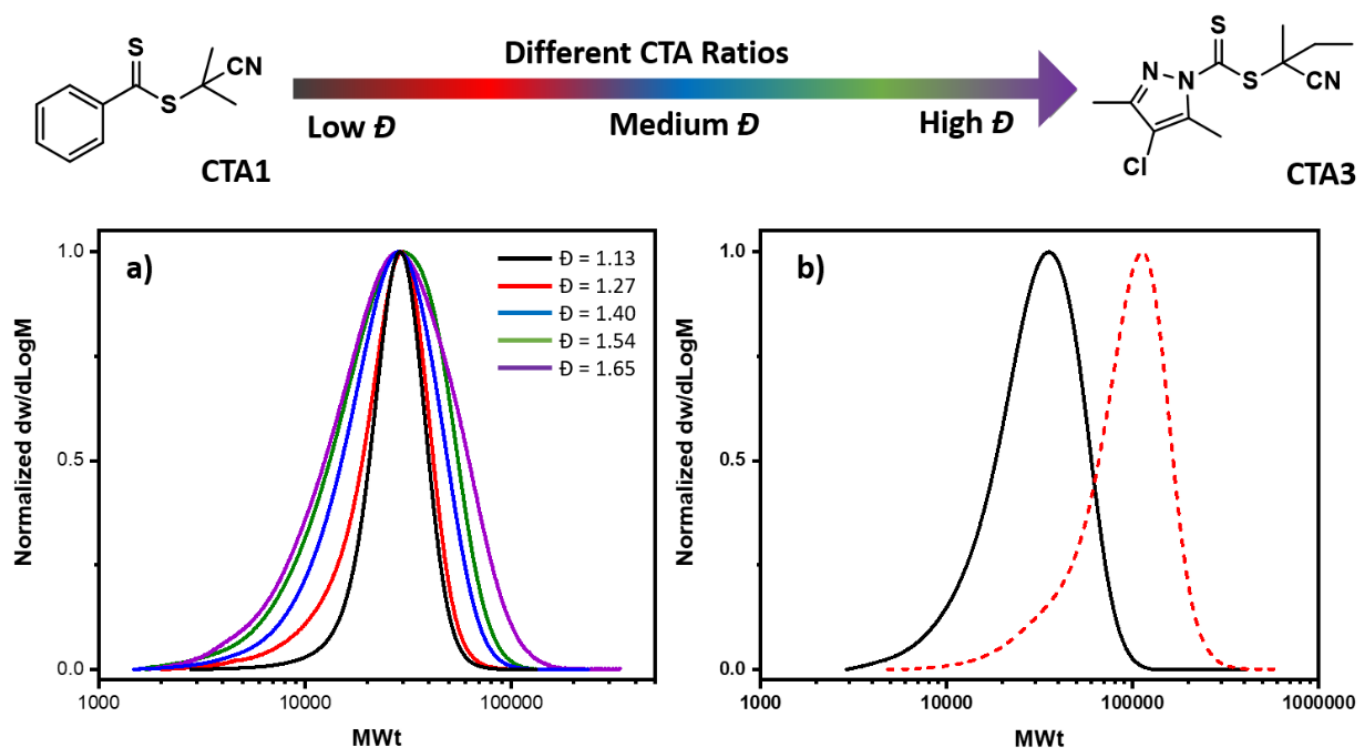

Fig. 2: SEC analysis of the polymerization of MMA, illustrating a) the variation in dispersity as CTA 1 and CTA 3 are mixed in different ratios and $b$ ) a chain extension of a PMMA macroCTA prepared with $35 \%$ CTA 1 and $65 \%$ CTA 3 with MMA.

With these results, it is worthwhile to have a closer investigation on how the two RAFT agents behave during the polymerization. We therefore simulated the reactions via deterministic kinetic modelling (see Supplementary Information for details), assuming ideal RAFT polymerization schemes and typical parameters for an AIBN-initiated MMA 
polymerization. In the model, the molar fraction between the efficient (high transfer) RAFT agent CTAx and the less efficient (low transfer) RAFT agent CTAy was varied. The transfer rate of the efficient RAFT agent has little influence on the outcome of the experiments, as long as it is sufficiently high (at least equal to the macroradical propagation rate, hence when $\left.k_{\mathrm{p}} \cdot c_{\mathrm{M}} \cdot c_{\mathrm{R}} \leq k_{\mathrm{ad}} \cdot c_{\mathrm{RAFT}^{*}} c_{\mathrm{R}}\right)$. This is not surprising and a common condition of RAFT polymerization. The low-efficiency RAFT agent has stricter kinetic boundaries. If the transfer rate (or macroradical addition rate to the CTA in that respect) is equally high, no larger dispersities can be obtained. If it is too low, then the less efficient RAFT agent becomes a bystander in the reaction and does not interact sufficiently with the radicals. Modelling showed that best results are obtained when the transfer constant of the less efficient RAFT agent is around 1-2 (which is expressed by almost equal RAFT addition and propagation rate coefficients). Altogether the simulations are fully in line with the experimental results, as can be seen in Fig. 3, which shows that by using a judiciously selected mixture of 2 suitable RAFT agents, the dispersity can be controlled upon demand. The dispersity changes slightly throughout the polymerization, and reaches distinct levels depending on the mole fraction of CTAs used. This is also nicely visible in the broadening of the simulated molecular weight distributions. Interestingly, the more efficient RAFT agent will always govern the process at the beginning of the reaction. Since the addition rate is high, the good RAFT agent is converted quickly and enters the main equilibrium. In opposition, the less efficient RAFT agent acts more like a conventional (non-degenerative) chain transfer agent, and merely captures macroradicals and blocks their further growth. This leads to the overall broad distributions right from the start of the polymerization. Only when all of CTAy (the less efficient one) is used up, or removed via purification, this RAFT agent can fully enter the main equilibrium allowing for chain growth. This explains why block extensions are still perfectly possible (a simulation of a block extension for a mole fraction of $x=0.5$ is shown in the insert of Fig. 3) despite the mechanistic 
difference in the homopolymerization. As for any RAFT process, the chain end fidelity is very high, and reflects the ratio between the efficient RAFT agent concentration and the amount of less efficient RAFT agent converted. The simulations show qualitatively similar results when repeating them for different monomer classes and propagation rates. The same dependencies as described above are visible, and seem to apply almost universally with only the chain transfer constant of the less efficient RAFT agent being important.
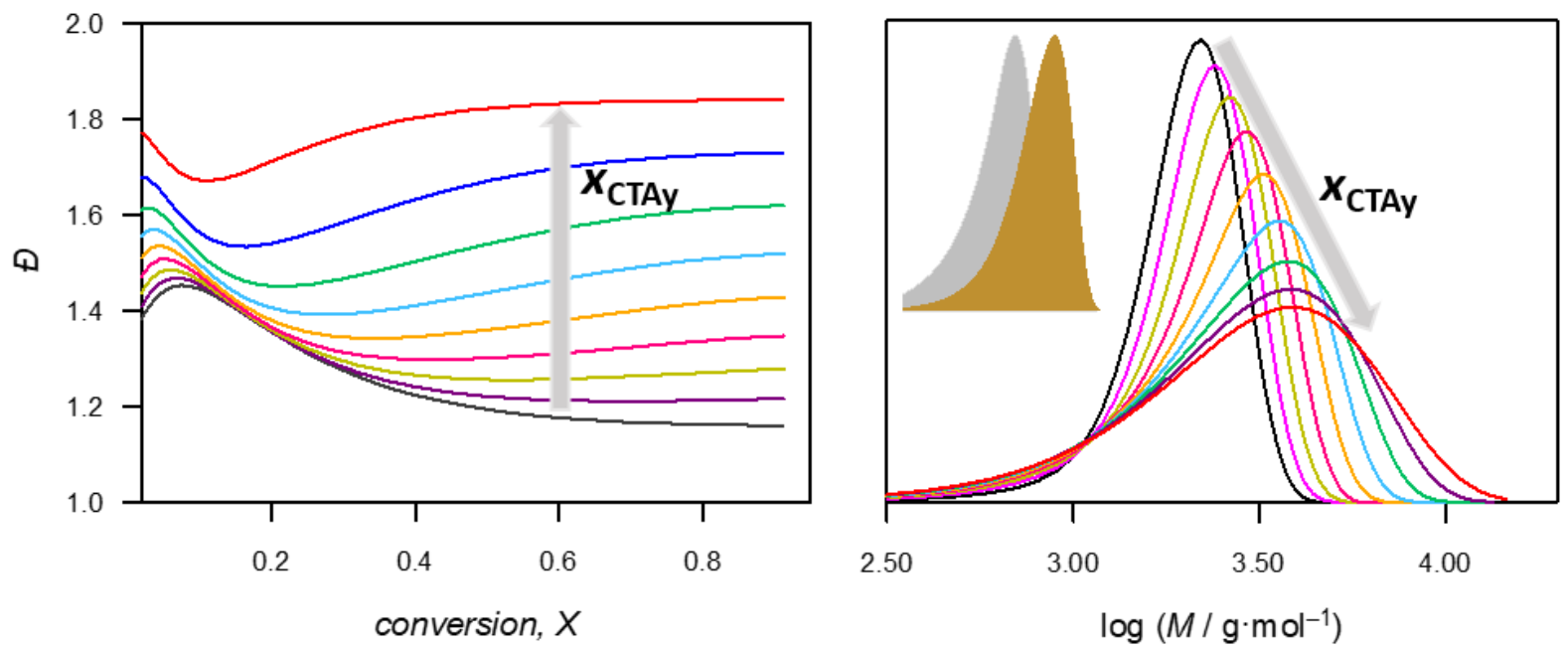

Fig. 3: Simulation of overall polymer dispersity as a function of monomer conversion (left) and molecular weight distributions at high conversion for $k_{\mathrm{ad}, \mathrm{CT} \mathrm{A}_{-} \mathrm{y}}=k_{\mathrm{p}}$ with increasing mole fractions of the less efficient RAFT agent CTAy (right). The insert shows the simulation result for a block extension from polymer obtained for [CTAx]=[CTAy].

Our strategy can also be extended to other methacrylic monomers (e.g. butyl and benzyl methacrylate, Supplementary Fig. 7-9 and Supplementary Table 5). Only methacrylic acid (MAA), a particularly challenging monomer for other CRP methodologies, poses larger problems within the methacrylate monomer family. ${ }^{50,51}$ The use of any mixture of CTA 1 and CTA 3 gave rise to bimodal MWDs prior to gelation, possibly attributed to the high polymerization rate of methacrylic acid (Supplementary Fig. 10). We therefore used a CTA with higher reactivity than CTA 3 for maintaining the controlled polymerization of methacrylic acid (Fig. 4). Indeed, upon substituting CTA 3 with CTA 2, the $\oslash$ of methacrylic acid could be 
efficiently tuned from 1.13 to 1.52 with monomodal MWDs achieved in all cases (Fig. 5d, Supplementary Fig. 11 and Supplementary Table 6).

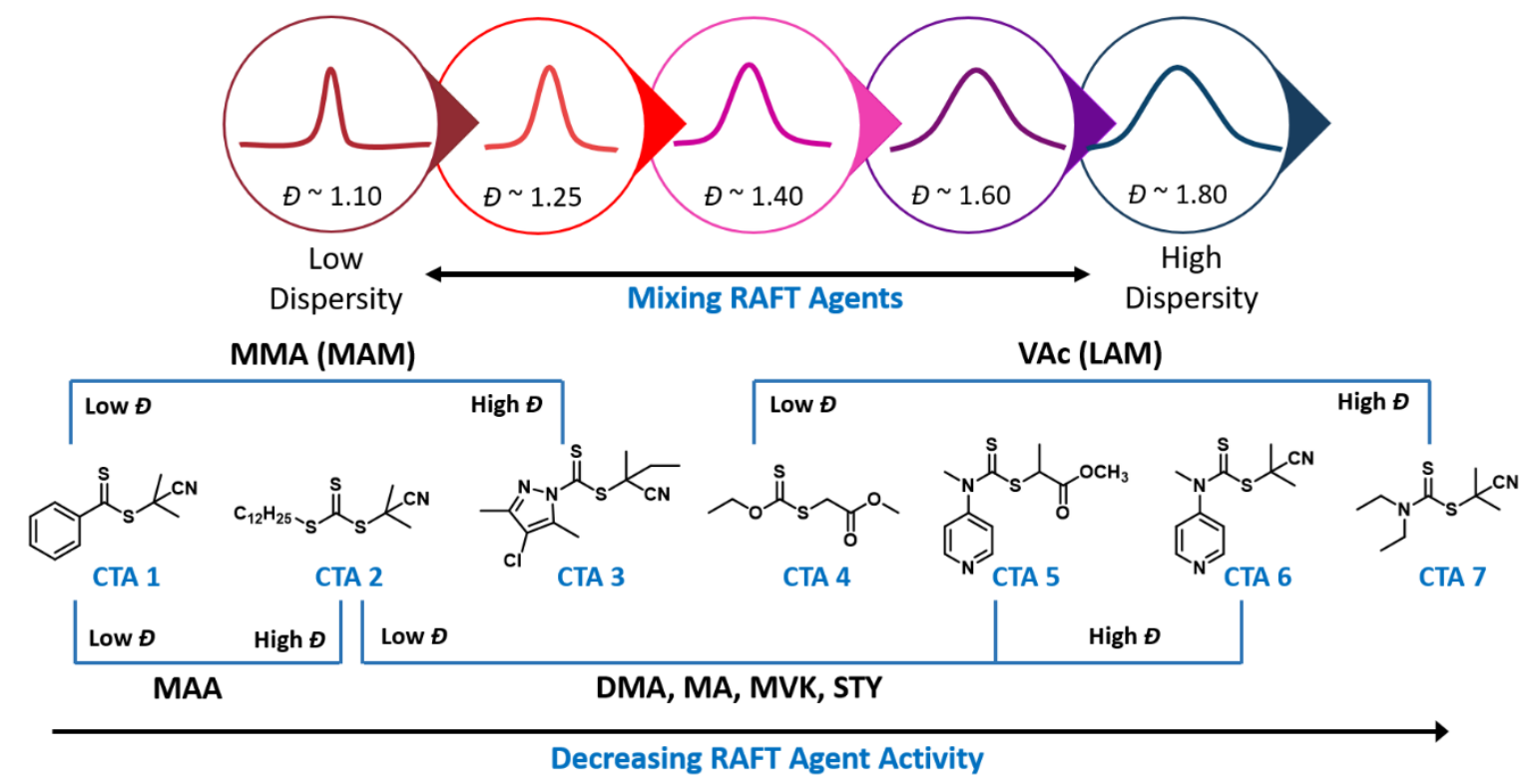

Fig. 4. Schematic representation of the RAFT chain transfer agents that are mixed to tune $\oslash$ of 6 different monomer classes.

To further expand the scope of our strategy in controlling the $\oslash$ of other polymer classes, methyl acrylate (MA) was next used as a model acrylic monomer. It is worth noting that the use of CTA 1 led to retardation/inhibition of polymerization due to the high stability of intermediate radicals (Supplementary Table 7, Entry 1). However, trithiocarbonates (CTA 2) have been reported to control the polymerization of acrylates very well and gave rise to very low dispersity PMA $(\nexists \sim$ 1.08, Fig. 5a, Supplementary Fig. 12 and 13a and Supplementary Table 7, Entry 2). As expected, the use of methyl 2-(butylthiocarbonothioylthio)propanoate, 2cyanobutan-2-yl 3,5-dimethyl-1H-pyrazole-1-carbodithioate and CTA 3 also yielded welldefined polymers with a final $Ð$ of 1.09, 1.10 and 1.11, respectively (Supplementary Fig. 13bd and Supplementary Table 7, Entries 3-5), thus suggesting that they can also be employed as alternatives for RAFT CTAs with high activity toward acrylates (in addition to CTA 2). Finding a suitable low activity RAFT agent is challenging for acrylates, as most RAFT agents show high chain transfer activity towards them due to the secondary radical nature of the propagating 
chains. Therefore, we selected CTA 5 and CTA 6, which both feature the same Z group but different R groups (Fig. 4). Pleasingly, both CTAs yielded PMA with the desirable broad MWDs ( $Ð \sim 1.63$ for CTA 5 and 1.74 for CTA 6, Supplementary Fig. 13e and Supplementary Table 7, Entry 7 \& Table 7, Entry 1) while also demonstrating monomodal SEC traces and can thus be used interchangeably as low-activity RAFT CTAs. Similar to PMMA, selecting a much lower activity CTA resulted in bimodal PMA with a significant low molecular weight shoulder and low RAFT efficiency (Supplementary Fig. 13f and Supplementary Table 7, Entry 6), which highlights the importance of selecting suitable low-activity RAFT CTAs. To successfully tune $Ð$ for PMA, we thus chose to mix CTA 2 with CTA 6 , and by changing the ratio of these two CTAs, polymers of $Ð \sim 1.09,1.25,1.37$ and 1.63 could be prepared (Fig. 4 and 5a). Again, monomodal MWDs were obtained revealing the compatibility of our approach with polyacrylates.

\section{Common Polymer Classes}
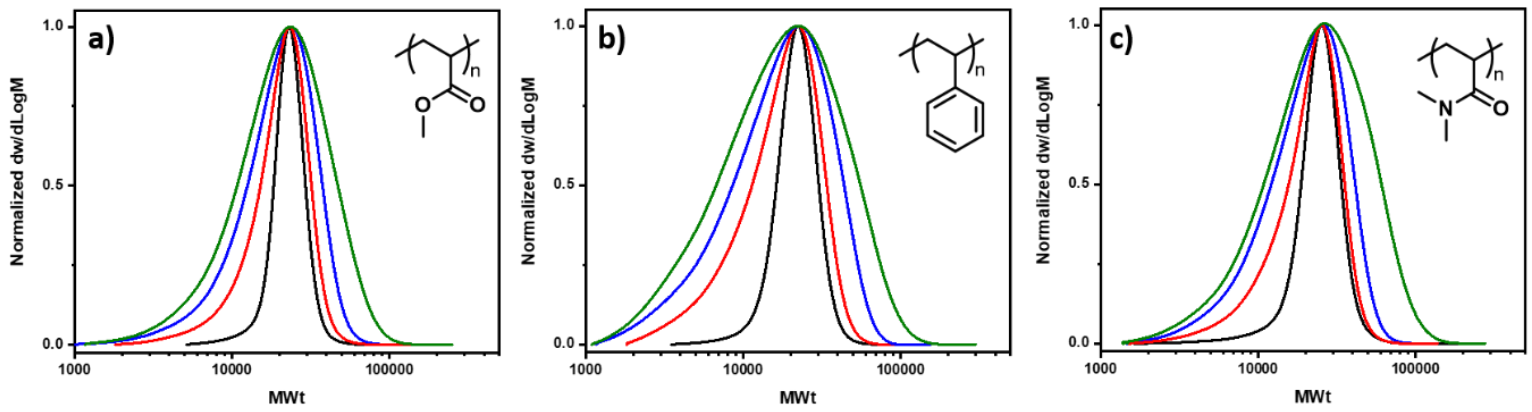

Challenging Polymer Classes
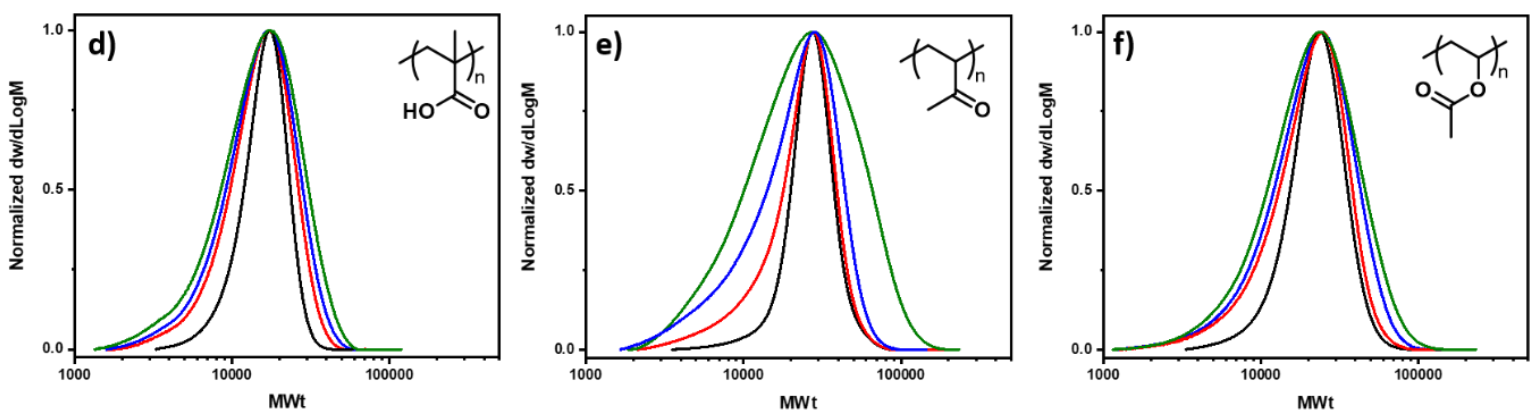

Fig. 5: SEC analysis of the polymerization of a) methyl acrylate (CTAs 2 and 6 mixed), b) styrene (CTA 2 and 6), c) dimethyl acrylamide (CTA 2 and 5) d) methacrylic acid (CTA 1 and 2) e) methyl vinyl ketone (CTA 2 and 6) and f) vinyl acetate (CTA 4 and 7), illustrating the variation in dispersity as two CTAs are mixed in different ratios. 
In addition, the $D$ of polystyrene could also be varied between 1.09 and 2.10 by employing mixtures of the same CTAs (CTA 2 + CTA 6, Fig. 4, Fig. 5b, Supplementary Fig. 14 and Supplementary Table 9). The same combination was also found to be adequate to control $Ð$ of poly(methyl vinyl ketone), another challenging material to synthesize by other CRP methods such as ATRP (Fig. 5e, Supplementary Fig. 15 and Supplementary Table 10). ${ }^{52}$ For the case of polyacrylamides, dimethyl acrylamide could be efficiently polymerized using mixtures of either CTA 2 and CTA 5, or CTA 2 and CTA 6, yielding a broad range of $Ð$ from 1.15 to 1.70 (Fig. 4, Fig. 5c, Supplementary Fig. 16 and Supplementary Table 11). To further probe the potential of our approach to control the $\oslash$ of even low-activated monomers, vinyl acetate was chosen. In that case, methyl (ethoxycarbonothioyl)sulfanyl acetate (CTA 4) was selected as high-activity CTA and 1-cyano-1-methylethyldiethyldithiocarbamate (CTA 7) as the low-activity CTA. Different ratios allowed tailoring of the MWDs with $Ð \sim 1.20-1.60$ (Fig. 5f, Supplementary Fig. 17 and Supplementary Table 12). For all 6 different monomer families, monomodal MWDs and very high RAFT efficiency were observed. Altogether, these results not only demonstrate the versatile nature of this new strategy, but also provide guidelines for the judicious selection of suitable RAFT agents to conveniently tune the dispersity of a variety of polymer classes.

To investigate whether high end-group fidelity was maintained, the preparation of a range of diblock copolymers were attempted. In particular, PDMA macroCTA obtained by mixing CTA $2+$ CTA $5\left(Đ \sim 1.53, M_{\mathrm{n}}=13000 \mathrm{~g} \mathrm{~mol}^{-1}\right)$ was chain extended with hydroxyethyl acrylamide (HEAm), yielding a P(MA- $b$-HEAm) diblock copolymer with an obvious shift to very high molecular weights (final $M_{\mathrm{n}}=104,500 \mathrm{~g} \mathrm{~mol}^{-1}, Ð \sim 1.65$, Fig. 6a, Supplementary Fig. 18 and Supplementary Table S13). In a similar vein, starting from a PMMA macroCTA obtained by mixing CTA $1+$ CTA $3\left(\nexists \sim 1.38, M_{\mathrm{n}}=24600 \mathrm{~g} \mathrm{~mol}^{-1}\right)$, a nicely chain extended P(MMA- $b$-BMA) diblock was evident by SEC (final $M_{\mathrm{n}}=64.700 \mathrm{~g} \mathrm{~mol}^{-1}, Ð \sim 1.43$, Fig. 6b, 
Supplementary Fig. 19 and Supplementary Table 13). Although it is not common to employ low-activity CTAs for block copolymers, we were also interested in assessing the end-group fidelity when starting from our highest $Ð$ polymers, which were synthesized by the exclusive use of a CTA with low reactivity. Impressively, by using a PMA macroCTA obtained by using only CTA $6\left(\nexists \sim 1.72, M_{\mathrm{n}}=24700 \mathrm{~g} \mathrm{~mol}^{-1}\right)$, a successful chain extension with DMA could be performed $\left(D \sim 1.71, M_{\mathrm{n}}=53600 \mathrm{~g} \mathrm{~mol}^{-1}\right.$, Fig. 6c, Supplementary Fig. 20 and Supplementary Table 14). These results are also in agreement with the simulations discussed above. Similarly, by utilizing a high $Ð$ PMMA macroCTA made exclusively using CTA 5, well-defined PMMAb-PVK (Fig. 6d, Supplementary Fig. 21 and Supplementary Table 14) and PMMA-b-PMAA block copolymers (Supplementary Fig. 22-23) were obtained. It is noted that in all cases, monomodal MWDs were maintained for the second block. The combination of this data strongly supports that polymers with tuneable dispersity and high-end group fidelity while exhibiting monomodal MWDs and high RAFT efficiency can be obtained when suitable CTAs were carefully selected, with the choice of the low-activity CTA being of particular importance. 

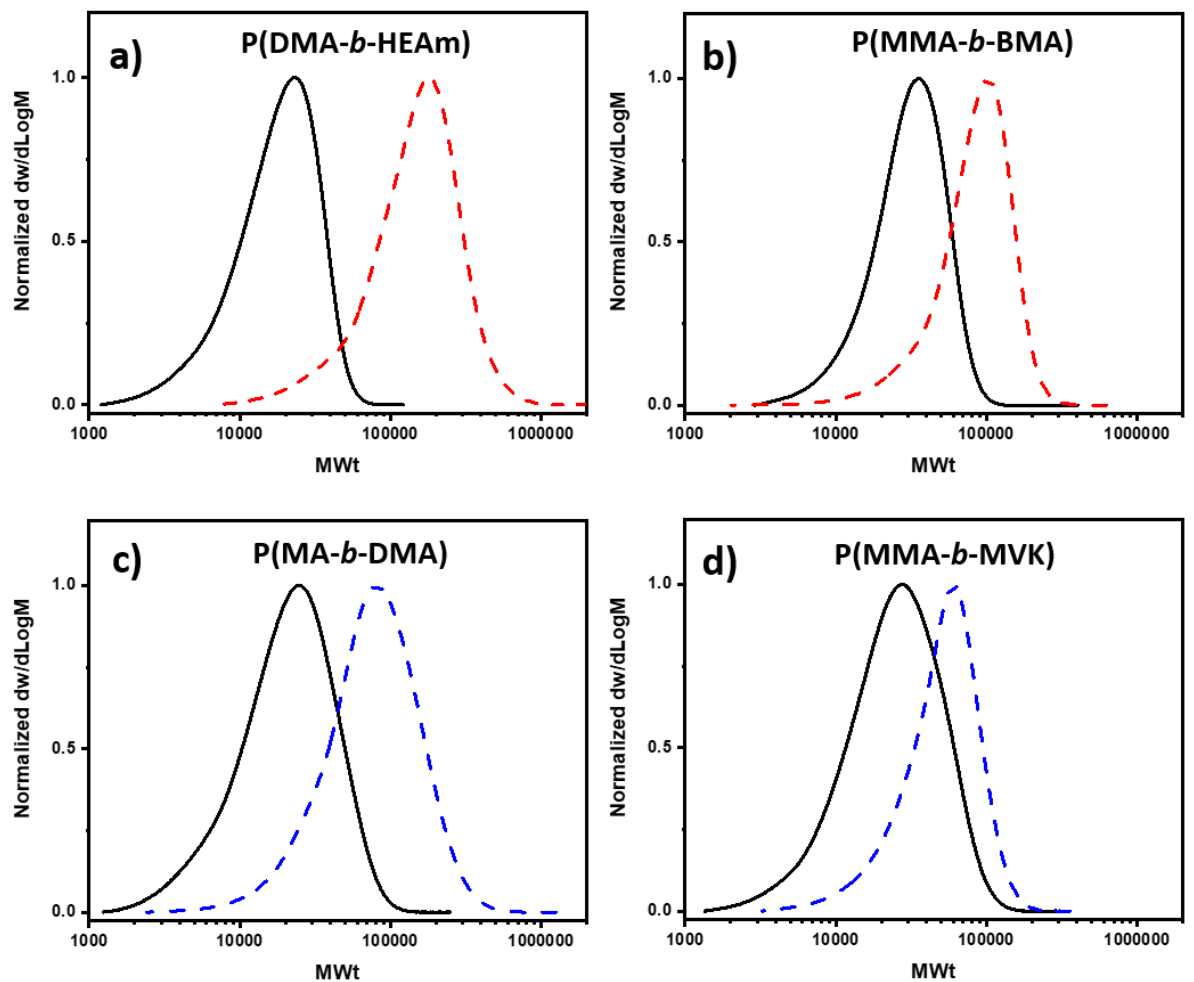

Fig. 6: SEC analysis of block copolymers formed from mixed RAFT agents for a) P(DMA- $b$-PHEAm) and b) P(MMA- $b$-PBMA) and formed from the low-activity CTA for c) P(MA- $b$-DMA) and d) $\mathrm{P}(\mathrm{MMA}-b-\mathrm{MVK})$.

\section{CONCLUSIONS}

To summarize, we report a facile and versatile RAFT polymerization strategy to tailor the $D$ of a range of polymer classes, encompassing polymethacrylates (including poly(methacrylic acid)), polyacrylates, polyacrylamides, polystyrene, poly(vinyl ketone) and poly(vinyl acetate). Key to our approach is the judicious selection and subsequent mixing of RAFT agents with different (yet suitable) reactivities, which allow for a wide range of dispersities to be obtained $(\nexists \sim 1.08-2.10)$ while exhibiting monomodal SEC traces and high RAFT efficiencies. Even for the high $\oslash$ obtained, excellent end-group fidelity could be demonstrated, as shown by efficient block copolymer formation. All results are fully in agreement with simulation data which further support our methodology. The versatile and robust nature of our methodology combined with the ready availability of the RAFT CTAs significantly expands the accessibility of polymeric materials with tuneable $\oslash$, especially to 
those challenging to access by other techniques. This work also provides a useful guideline for the judicious selection of RAFT agents for tuning polymer $\oslash$, which may attract broad interest in polymer community and beyond.

\section{EXPERIMENTAL PROCEDURES}

\section{General Procedure: PMMA $(\boxplus=1.13)$}

Into a $4 \mathrm{~mL}$ glass vial, $5.17 \mathrm{mg}$ of 2-cyanoprop-2-yl dithiobenzoate (CTA 1, 1 equiv.) were dissolved in $0.9 \mathrm{~mL}$ of DMF. A stock solution of AIBN $(3.9 \mathrm{mg})$ was prepared in $1.02 \mathrm{~mL}$ of DMF, and $100 \mu \mathrm{L}$ of this solution $(0.383 \mathrm{mg}, 0.1$ equiv.) were transferred to the vial. Subsequently, $0.75 \mathrm{~mL}$ of methyl methacrylate (300 equiv.) and a stirrer bar were added, and the vial was sealed with a septum, prior to deoxygenation by nitrogen bubbling for 15 minutes. Polymerization was conducted in an oil bath at $70{ }^{\circ} \mathrm{C}$ for 22 hours with a $200 \mathrm{rpm}$ stirring rate. Samples were taken periodically under a nitrogen blanket for ${ }^{1} \mathrm{H}$ NMR analysis and passed through a syringe filter $(0.45 \mu \mathrm{M}$ PTFE membrane) prior to SEC analysis.

\section{General Procedure: PMMA $(\oplus=1.65)$}

Into a $4 \mathrm{~mL}$ glass $10.1 \mathrm{mg}$ of 2-cyanobutan-2-yl 3,5-dimethyl-1H-pyrazole-1-carbodithioate (CTA 3, 1 equiv.) were dissolved in $0.9 \mathrm{~mL}$ of DMF. A stock solution of AIBN (4.2 mg) was prepared in $0.73 \mathrm{~mL}$ of DMF, and $100 \mu \mathrm{L}$ of this solution ( $0.58 \mathrm{mg}, 0.1$ equiv.) were transferred to the vial. Subsequently, $0.75 \mathrm{~mL}$ of methyl methacrylate (200 equiv.) and a stirrer bar were added, and the vial was sealed with a septum, prior to deoxygenation by nitrogen bubbling for 15 minutes. Polymerization was conducted in an oil bath at $70^{\circ} \mathrm{C}$ for 22 hours with a $200 \mathrm{rpm}$ stirring rate. Samples were taken periodically under a nitrogen blanket for ${ }^{1} \mathrm{H}$ NMR analysis and passed through a syringe filter $(0.45 \mu \mathrm{M}$ PTFE membrane) prior to SEC analysis.

\section{General Procedure: PMMA $(\oplus=1.27,1.40,1.54)$}

Procedure 1.2 was repeated with mixtures of 2-cyanoprop-2-yl dithiobenzoate (CTA 1) and 2cyanobutan-2-yl 3,5-dimethyl-1H-pyrazole-1-carbodithioate (CTA 3). Molar ratios containing 
60\% (4.65 mg CTA 1: 4.04 mg CTA 3), 35\% (2.72 mg CTA 1: 6.60 mg CTA 3) and 10\% (0.78

mg CTA 1: 9.08 mg CTA 3) CTA 1 yielded dispersities of 1.27, 1.40 and 1.54 respectively.

\section{DATA AND SOFTWARE AVAILABILITY}

All relevant data is available from the authors.

\section{SUPPLEMENTAL INFORMATION}

Please see the supplementary information for detailed experimental procedures along with 2 supplementary schemes, 12 supplementary tables and 25 supplementary figures.

\section{ACKNOWLEDGEMENTS}

A.A. gratefully acknowledges ETH Zurich for financial support. N.P.T. acknowledges the award of a DECRA Fellowship from the ARC (DE180100076). We acknowledge Thomas Congdon, Daniel Messmer and Manon Rolland for scientific discussions.

\section{AUTHOR CONTRIBUTIONS}

A.A. conceived the initial idea and managed the overall project. R.W., A.A. and N.P.T. designed the experiments. R.W. performed the vast majority of the experiments and analyzed the data with input from K.P., N.P.T. and A.A.. K.P. conducted the polyacrylamide experiments. T.J. carried out all modelling of polymerizations. R.W., and A.A. co-wrote the manuscript with input from N.P.T and T.J.. T.J. wrote the simulation part. All authors discussed the results and commented on the manuscript.

\section{DECLARATION OF INTERESTS}

The authors declare no competing financial interest.

\section{REFERENCES}

1. Moad, G., Rizzardo, E., and Thang, S.H. (2012). Living radical polymerization by the RAFT processa third update. Aust. J. Chem. 65, 985-1076.

2. Matyjaszewski, K., and Tsarevsky, N.V. (2009). Nanostructured functional materials prepared by atom transfer radical polymerization. Nat. Chem. 1, 276.

3. Pan, X., Tasdelen, M.A., Laun, J., Junkers, T., Yagci, Y., and Matyjaszewski, K. (2016). Photomediated controlled radical polymerization. Prog. Polym. Sci. 62, 73-125. 
4. Bates, C.M., and Bates, F.S. (2017). 50th Anniversary Perspective: Block Polymers - Pure Potential. Macromolecules 50, 3-22.

5. Moad, G., Rizzardo, E., and Thang, S.H. (2008). Radical addition-fragmentation chemistry in polymer synthesis. Polymer 49, 1079-1131.

6. Barner-Kowollik, C. (2008). Handbook of RAFT polymerization (Weinheim, Germany: John Wiley \& Sons).

7. Perrier, S. (2017). 50th Anniversary Perspective: RAFT Polymerization - A User Guide. Macromolecules 50, 7433-7447.

8. Matyjaszewski, K. (2012). Atom transfer radical polymerization (ATRP): current status and future perspectives. Macromolecules 45, 4015-4039.

9. Boyer, C., Bulmus, V., Davis, T.P., Ladmiral, V., Liu, J., and Perrier, S. (2009). Bioapplications of RAFT polymerization. Chem. Rev. 109, 5402-5436.

10. Destarac, M. (2010). Controlled radical polymerization: industrial stakes, obstacles and achievements. Macromol. React. Eng. 4, 165-179.

11. Siegwart, D.J., Oh, J.K., and Matyjaszewski, K. (2012). ATRP in the design of functional materials for biomedical applications. Prog. Polym. Sci. 37, 18-37.

12. Destarac, M. (2018). Industrial development of reversible-deactivation radical polymerization: is the induction period over? Polym. Chem. 9, 4947-4967.

13. Gilbert, R., Hess, M., Jenkins, A., Jones, R., Kratochvil, P., and Stepto, R.F. (2009). Dispersity in polymer science. Pure Appl. Chem. 81, 351-353.

14. Stepto, R.F. (2009). Dispersity in polymer science (IUPAC Recommendations 2009). Pure Appl. Chem. 81, 351-353.

15. Mahabadi, H.K., and O'driscoll, K. (1977). Termination rate constant in free-radical polymerization. J. Polym. Sci. Polym. Chem. Ed. 15, 283-300.

16. Whitfield, R., Truong, N.P., Messmer, D., Parkatzidis, K., Rolland, M., and Anastasaki, A. (2019). Tailoring polymer dispersity and shape of molecular weight distributions: methods and applications. Chem. Sci. 10, 8724-8734.

17. Gentekos, D.T., Sifri, R.J., and Fors, B.P. (2019). Controlling polymer properties through the shape of the molecular-weight distribution. Nat. Rev. Mater., 1-14.

18. Stürzel, M., Mihan, S., and Mülhaupt, R. (2016). From multisite polymerization catalysis to sustainable materials and all-polyolefin composites. Chem. Rev. 116, 1398-1433.

19. Doncom, K.E., Blackman, L.D., Wright, D.B., Gibson, M.I., and O'Reilly, R.K. (2017). Dispersity effects in polymer self-assemblies: a matter of hierarchical control. Chem. Soc. Rev. 46, 4119-4134.

20. Lynd, N.A., Meuler, A.J., and Hillmyer, M.A. (2008). Polydispersity and block copolymer selfassembly. Prog. Polym. Sci. 33, 875-893.

21. Quirk, R.P., and Lee, B. (1992). Experimental criteria for living polymerizations. Polym. Int. 27, 359367.

22. Lynd, N.A., and Hillmyer, M.A. (2005). Influence of polydispersity on the self-assembly of diblock copolymers. Macromolecules 38, 8803-8810.

23. Aoki, Y., Li, L., Uchida, H., Kakiuchi, M., and Watanabe, H. (1998). Rheological images of poly (vinyl chloride) gels. 5. Effect of molecular weight distribution. Macromolecules 31, 7472-7478.

24. Terreau, O., Luo, L., and Eisenberg, A. (2003). Effect of poly (acrylic acid) block length distribution on polystyrene-b-poly (acrylic acid) aggregates in solution. 1. Vesicles. Langmuir 19, 5601-5607.

25. Rubens, M., and Junkers, T. (2019). Comprehensive control over molecular weight distributions through automated polymerizations. Polym. Chem. 10, 6315-6323.

26. Rubens, M., and Junkers, T. (2019). A predictive framework for mixing low dispersity polymer samples to design custom molecular weight distributions. Polym. Chem. 10, 5721-5725.

27. Matsushita, Y., Mori, K., Saguchi, R., Nakao, Y., Noda, I., and Nagasawa, M.J.M. (1990). Molecular weight dependence of lamellar domain spacing of diblock copolymers in bulk. Macromolecules 23, 4313-4316. 
28. Domanskyi, S., Gentekos, D.T., Privman, V., and Fors, B.P. (2020). Predictive design of polymer molecular weight distributions in anionic polymerization. Polym. Chem. 11, 326-336.

29. Gentekos, D.T., Dupuis, L.N., and Fors, B.P. (2016). Beyond dispersity: Deterministic control of polymer molecular weight distribution. J. Am. Chem. Soc. 138, 1848-1851.

30. Gentekos, D.T., Jia, J., Tirado, E.S., Barteau, K.P., Smilgies, D.-M., DiStasio Jr, R.A., and Fors, B.P. (2018). Exploiting molecular weight distribution shape to tune domain spacing in block copolymer thin films. J. Am. Chem. Soc. 140, 4639-4648.

31. Kottisch, V., Gentekos, D.T., and Fors, B.P. (2016). "Shaping" the Future of Molecular Weight Distributions in Anionic Polymerization. ACS Macro Lett. 5, 796-800.

32. Corrigan, N., Almasri, A., Taillades, W., Xu, J., and Boyer, C. (2017). Controlling molecular weight distributions through photoinduced flow polymerization. Macromolecules 50, 8438-8448.

33. Corrigan, N., Manahan, R., Lew, Z.T., Yeow, J., Xu, J., and Boyer, C. (2018). Copolymers with Controlled Molecular Weight Distributions and Compositional Gradients through Flow Polymerization. Macromolecules 51, 4553-4563.

34. Morsbach, J., Müller, A.H., Berger-Nicoletti, E., and Frey, H. (2016). Living polymer chains with predictable molecular weight and dispersity via carbanionic polymerization in continuous flow: Mixing rate as a key parameter. Macromolecules 49, 5043-5050.

35. Reis, M.H., Varner, T.P., and Leibfarth, F.A. (2019). The Influence of Residence Time Distribution on Continuous-Flow Polymerization. Macromolecules 52, 3551-3557.

36. Liu, X., Wang, C.G., and Goto, A. (2019). Polymer Dispersity Control by Organocatalyzed Living Radical Polymerization. Angew. Chem. 131, 5654-5659.

37. Liu, D., Sponza, A.D., Yang, D., and Chiu, M. (2019). Modulating Polymer Dispersity with Light: Cationic Polymerization of Vinyl Ethers Using Photochromic Initiators. Angew. Chem. 58, 1621016216.

38. Plichta, A., Zhong, M., Li, W., Elsen, A.M., and Matyjaszewski, K. (2012). Tuning dispersity in diblock copolymers using ARGET ATRP. Macromol. Chem. Phys. 213, 2659-2668.

39. Wang, Z., Yan, J., Liu, T., Wei, Q., Li, S., Olszewski, M., Wu, J., Sobieski, J., Fantin, M., and Bockstaller, M.R. (2019). Control of Dispersity and Grafting Density of Particle Brushes by Variation of ATRP Catalyst Concentration. ACS Macro Lett. 8, 859-864.

40. Whitfield, R., Parkatzidis, K., Rolland, M., Truong, N.P., and Anastasaki, A. (2019). Tuning Dispersity by Photoinduced Atom Transfer Radical Polymerisation: Monomodal Distributions with ppm Copper Concentration. Angew. Chem. Int. Ed. 58, 13323-13328.

41. Semsarilar, M., and Perrier, S. (2010). 'Green'reversible addition-fragmentation chain-transfer (RAFT) polymerization. Nat. Chem. 2, 811.

42. Barner, L., Davis, T.P., Stenzel, M.H., and Barner-Kowollik, C. (2007). Complex macromolecular architectures by reversible addition fragmentation chain transfer chemistry: theory and practice. Macromol. Rapid Comm. 28, 539-559.

43. Khor, S.Y., Vu, M.N., Pilkington, E.H., Johnston, A.P., Whittaker, M.R., Quinn, J.F., Truong, N.P., and Davis, T.P. (2018). Elucidating the Influences of Size, Surface Chemistry, and Dynamic Flow on Cellular Association of Nanoparticles Made by Polymerization-Induced Self-Assembly. Small 14, 1801702.

44. Ramírez-García, P.D., Retamal, J.S., Shenoy, P., Imlach, W., Sykes, M., Truong, N., Constandil, L., Pelissier, T., Nowell, C.J., and Khor, S.Y. (2019). A pH-responsive nanoparticle targets the neurokinin 1 receptor in endosomes to prevent chronic pain. Nat. Nanotechnol. 14, 1150-1159.

45. Mueller, A.H., Zhuang, R., Yan, D., and Litvinenko, G.J.M. (1995). Kinetic analysis of" living" polymerization processes exhibiting slow equilibria. 1. degenerative transfer (direct activity exchange between active and" dormant" species). application to group transfer polymerization. Macromolecules 28, 4326-4333.

46. Nejad, E.H., Castignolles, P., Gilbert, R.G., and Guillaneuf, Y. (2008). Synthesis of methacrylate derivatives oligomers by dithiobenzoate-RAFT-mediated polymerization. J. Polym. Sci. Pol. Chem. 46, 2277-2289. 
47. Truong, N.P., Quinn, J.F., Dussert, M.V., Sousa, N.B., Whittaker, M.R., and Davis, T.P. (2015). Reproducible access to tunable morphologies via the self-assembly of an amphiphilic diblock copolymer in water. ACS Macro Lett. 4, 381-386.

48. Derboven, P., D'hooge, D.R., Reyniers, M.-F., Marin, G.B., and Barner-Kowollik, C. (2015). The long and the short of radical polymerization. Macromolecules 48, 492-501.

49. Gardiner, J., Martinez-Botella, I., Kohl, T.M., Krstina, J., Moad, G., Tyrell, J.H., Coote, M.L., and Tsanaktsidis, J. (2017). 4-Halogeno-3, 5-dimethyl-1 H-pyrazole-1-carbodithioates: versatile reversible addition fragmentation chain transfer agents with broad applicability. Polym. Int. 66, 1438-1447.

50. Patten, T.E., and Matyjaszewski, K. (1998). Atom transfer radical polymerization and the synthesis of polymeric materials. Adv. Mater. 10, 901-915.

51. Fantin, M., Isse, A.A., Venzo, A., Gennaro, A., and Matyjaszewski, K. (2016). Atom transfer radical polymerization of methacrylic acid: a won challenge. J. Am. Chem. Soc. 138, 7216-7219.

52. Mittal, A., Sivaram, S., and Baskaran, D. (2006). Unfavorable coordination of copper with methyl vinyl ketone in atom transfer radical polymerization. Macromolecules 39, 5555-5558. 